\title{
Wearable Textile Antenna Magnetically Coupled to Flexible Active Electronic Circuits
}

\author{
M. Virili, H. Rogier, F. Alimenti, P. Mezzanotte, L. Roselli
}

\begin{abstract}
This paper proposes wearable electronic modules on textile, suitable for garment integration, based on antennas magnetically coupled to the active circuitry. The coupling mechanism is based on a heterogeneous transformer with the primary and secondary windings implemented in the antenna and in the circuitry substrate (hybrid or monolithic), respectively. The proposed coupling topology avoids galvanic contacts between the antenna and the active circuitry, allowing for interconnecting the antenna by a mere placing and gluing process. Bonding and soldering processes, often critical for textile materials are thus avoided. The proposed innovation, mitigating the industrial realization constraints posed by textile implementation of active antennas and RFID tags, should unsurprisingly enable and foster industrial developments of garment RFID, wearable electronics and body-centric communications systems. A patch antenna with transformer based on textile materials and operating in the frequency band 2.4-2.4835 GHz is designed, realized and measured.
\end{abstract}

Index Terms - Textile technology, flexible electronics, wearable electronics, RFID tags, antenna feeds, transformers, magnetic coupling, non-ohmic contacts interconnections, body centric communication, BAN.

\section{INTRODUCTION}

A utomatic identification procedures and wearable textile systems are, nowadays, attractive fields of research. The Radio Frequency IDentification (RFID) systems play an important role in automatic identification and monitoring procedures. RFID allows objects and their neighboring environment to become self-describing by communicating and monitoring their identity and relevant information. Wearable RFIDs and Smart Fabrics and Interactive Textiles (SFIT) rely on the unobtrusive integration of electronic systems into garments [1-5], ultimately enabling wearable intelligence. Intelligent wearable systems extend the functionality of professional garments (for instance the protection level) by providing more information about the wearer's operating conditions, such as health state and environmental parameters, by means of wearable sensors networks potentially integrated in Body Area Networks (BAN). Without lack of generality, in this letter we focus on firefighter and emergency disaster

This work is supported in part by the COST RFCSET IC0803 (http://www.cost-ic0803.org/).

M. Virili, F. Alimenti, P. Mezzanotte, and L. Roselli are with the Dept. Of Electronic and Information Engineering (DIEI) of the University of Perugia, Perugia, Italy, (corresponding author e-mail: marco.virili@diei.unipg.it).

H. Rogier is with the Dept. of Information Technology (INTEC) of Gent University, Gent, Belgium. personnel garments [2].

SFIT systems as well as wearable RFIDs require that active electronic circuits (sensors and control units) transmit and receive wirelessly via antenna systems. Generally, these subsystems, antennas and circuits, are directly interconnected by means of galvanic contacts, thus requiring a wire-bonding or a soldering process. Recently a novel method to connect such subsystems has been proposed in [5-10]. This method is based on magnetic coupling and promises to enhance the integration level of wearable electronic systems. In particular, for the simple yet non-restrictive antenna-chip case, a transformer performs the coupling by means of two windings realized on the antenna and electronic substrates, respectively. As a result, the chip (or the hybrid circuit) can be mounted by a mere placing and gluing process [11]. In this way, the chip is pad-less and completely passivated; bonding and soldering processes, critical for textile materials, are avoided. It is worth noticing that this feeding topology works similarly to aperture coupling [4] (the coupling is provided without direct contact) but in this case a lumped element is used. The advantage of this approach is the compactness of the transformer, fundamental for low frequency application and an on-chip implementation [8]; the transformer dimensions are smaller than distributed elements like the aperture that is proportional to the wavelength.

The antenna-transformer prototype has been designed, fabricated and measured. The transformer's primary winding has been integrated onto antenna and implemented on the same textile substrate without loss of generality. The secondary winding has been realized on a flexible substrate for test purposes. The design of the antenna and the transformer aims at maximizing the power transfer between the antenna and the load (emulating the chip in the test application) connected to the secondary winding. The first step is the design and the optimization of the transformer. Next, a patch antenna is designed. The antenna is matched to the transformer optimum impedance, resulting in a transmission coefficient close to the Maximum Available Gain (MAG). Finally, the prototype is measured and the results are compared with the simulations.

\section{TRANSFORMER AND ANTENNA DESIGN}

The antenna and the one-turn primary winding are realized on textile materials (Flectron and black foam) [12]. The oneturn secondary winding is realized on a flexible substrate (DuPont Pyralux) [13] and its terminals are connected to a 
UFL connector, necessary to characterize the antennatransformer system by means of the Vector Network Analyzer (VNA). The properties of the materials used are listed in Table I. The conductivity of Flectron was estimated from the sheet resistance (nominal value $\leq 0.07 \Omega / \square$ ), assuming a nominal thickness of $40 \mu \mathrm{m}$ and rounding the conductivity value for the worst case scenario. The material stack-up, starting from the bottom layer, is:

1. copper (UFL connector pads);

2. Pyralux (chip/circuitry substrate);

3. copper (secondary winding)

4. bi-adhesive tape (insulator between windings);

5. Flectron (antenna ground plane and primary winding);

6. Black foam (antenna substrate);

7. Flectron (patch antenna);

TABLE I

MATERIALS PROPERTIES

\begin{tabular}{ccccc}
\hline \hline Materials & $\begin{array}{c}\text { Thickness } \\
{[\mu \mathrm{m}]}\end{array}$ & $\begin{array}{c}\text { Permittivity } \\
\varepsilon_{\mathrm{R}}\end{array}$ & $\begin{array}{c}\text { Conductivity } \\
\sigma[\mathrm{S} / \mathrm{m}]\end{array}$ & $\begin{array}{c}\text { Loss } \\
\text { tangent }\end{array}$ \\
\hline Bi-adhesive & 100 & 3 & - & 0 \\
tape & 3940 & 1.575 & - & 0.024 \\
Black foam & 18 & - & $5.8 \cdot 10^{7}$ & - \\
Copper & 40 & - & $3 \cdot 10^{5}$ & - \\
Flectron & 50 & 3.2 & - & 0.001 \\
Pyralux & & & &
\end{tabular}

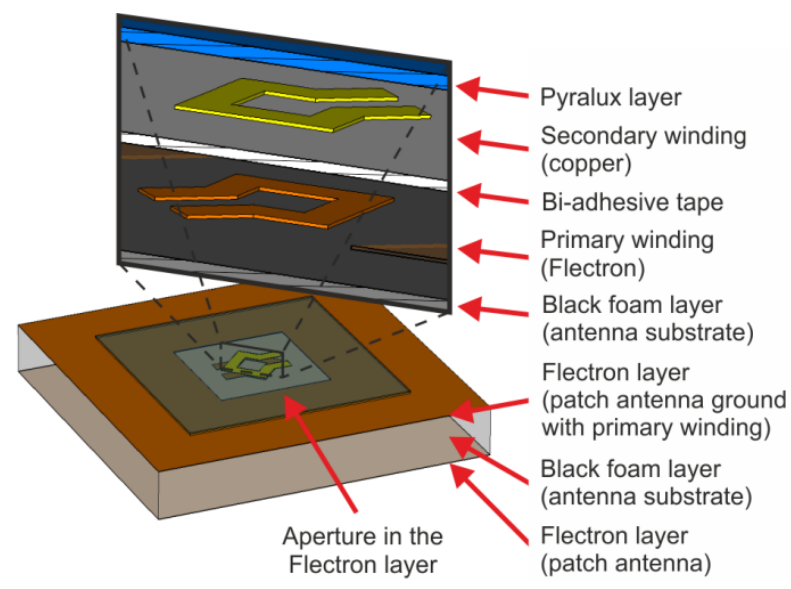

Fig. 1. Heterogeneous transformer layout with exploded view vertically sectioned: the side length of the square aperture on the Flectron is $10 \mathrm{~mm}$, the number of windings turns is 1 , the windings track width is $1 \mathrm{~mm}$, their inner radius is $1 \mathrm{~mm}$, the thicknesses of the primary Flectron winding and secondary copper winding are $40 \mu \mathrm{m}$ and $18 \mu \mathrm{m}$, respectively, and their distance is about $100 \mu \mathrm{m}$ (bi-adhesive tape thickness). In the exploded view the copper layer with the UFL connector over of the Pyralux and the patch antenna Flectron layer under the black foam are not shown.

\section{A. Transformer design}

The first step consists of designing the transformer. The transformer is inherently heterogeneous because the materials and the dimensions are, in general, different for the two windings. The two one-turn windings have an inner radius of 1 $\mathrm{mm}$ and a track width of $1 \mathrm{~mm}$. The transformer dimensions result from a compromise between the performance (transmission coefficient), the reliability and the technological constraints imposed by the fabrication process (design rules). The primary Flectron winding on the black foam is implemented in a square aperture (side $10 \mathrm{~mm}$ long) on the antenna ground plane. The secondary copper winding is realized on a small flexible Pyralux substrate with a standard photolithographic process, enabling a reliable interconnection with active electronic circuits and in the meanwhile maintaining flexibility and wearability of the module. A sheet of bi-adhesive tape separates both windings.

The transformer layout, shown in Fig. 1 and without antenna and UFL connector, was simulated with an EM simulator in order to find the optimum dimensions. The MAG of the heterogeneous transformer, in the case of simultaneous conjugate matching, is equal to $-0.27 \mathrm{~dB}$. At $2.45 \mathrm{GHz}$ the source (the antenna) and the load (the chip) optimum impedances of the transformer are found to be $Z_{S O P T}=88.3-\mathrm{j} 45.4 \Omega$ and $Z_{L O P T}=80-\mathrm{j} 103.2 \Omega$, respectively.

\section{B. Antenna design}

Once the optimal termination impedances of the transformer are known, the following step consists of designing the antenna. The antenna operates in the Industrial, Scientific, and Medical (ISM) frequency band for Short Range Devices (SRD) $(2.4 \div 2.4835 \mathrm{GHz})$. The patch antenna topology has been chosen because the antenna ground plane shields the body from antenna radiation and stabilizes the antenna characteristics in proximity of the human body, thereby mitigating the adverse effects that typically occur in bodycentric communication applications [2].

Computer-aided optimization by means of full-wave simulations has been performed to match the antenna impedance to the optimum source impedance of the transformer by varying the feeding point position, as in $[2,3]$. This procedure yields the maximum power transfer over the transformer to be obtained throughout the desired bandwidth. The simulation results in Fig. 2 refer to a square patch with a length of $44 \mathrm{~mm}$. The input impedance is equal to 93.23-j43.17 $\Omega$, quite close to the transformer optimum input impedance $88.3-\mathrm{j} 45.4 \Omega$ required by the transformer. The radiation efficiency is $-1.74 \mathrm{~dB}(67.5 \%)$ and the maximum gain is $6.71 \mathrm{dBi}$.

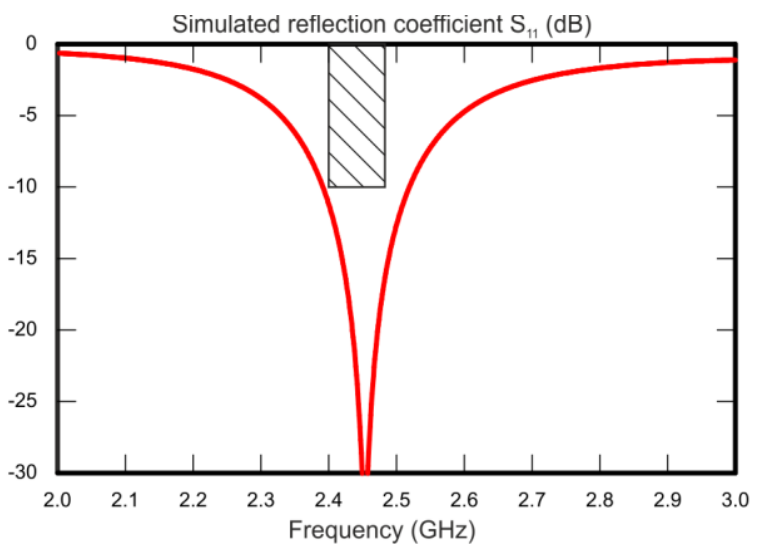

Fig. 2. Optimized patch antenna: reflection coefficient $S_{11}$. Reflection is computed with respect to the complex conjugate source impedance of the transformer $\left(Z_{\mathrm{S}_{-} \mathrm{OPT}} *=88.3+\mathrm{j} 45.4 \Omega\right)$.

\section{Sensitivity analysis}

A simulation campaign of the structure antenna-transformer 
was performed in order to analyze the effects of the distance (tape thickness) and horizontal misalignment variations of the windings. The simulations with horizontal misalignment were performed assuming a constant windings distance equal to 100 $\mu \mathrm{m}$ (nominal tape thickness). The radiation efficiency for a fixed input impedance is computed as

$$
\left.\eta_{Z}\right|_{d B}=\left.\sqrt{1-\left|S_{11}\right|^{2}}\right|_{d B}+\left.\eta\right|_{d B}
$$

where $\eta_{Z}$ is the radiation efficiency that takes into account the reflections introduced by the mismatch with the port impedance, $\eta$ is the radiation efficiency (ratio between the total radiated power and the power delivered to the antenna), and the term under the radical operator is the power delivered from the port to the system.

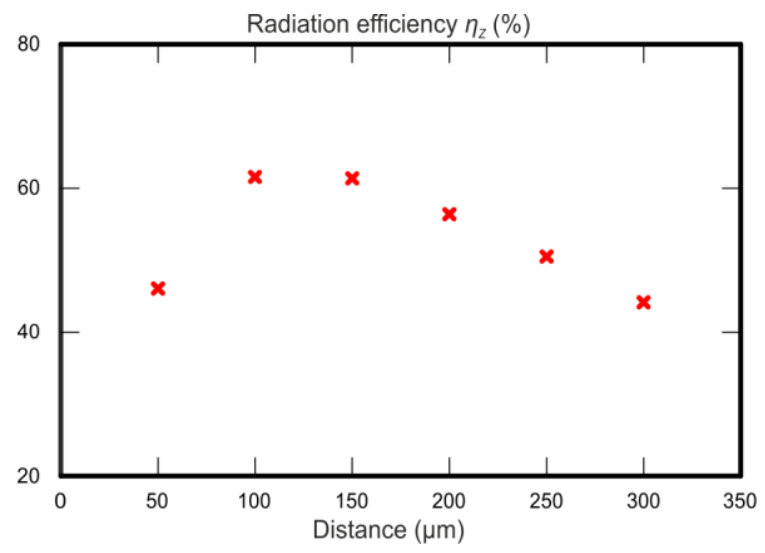

Fig. 3. Radiation efficiency of the transformer-antenna system varying the distance (tape thickness) between the windings.

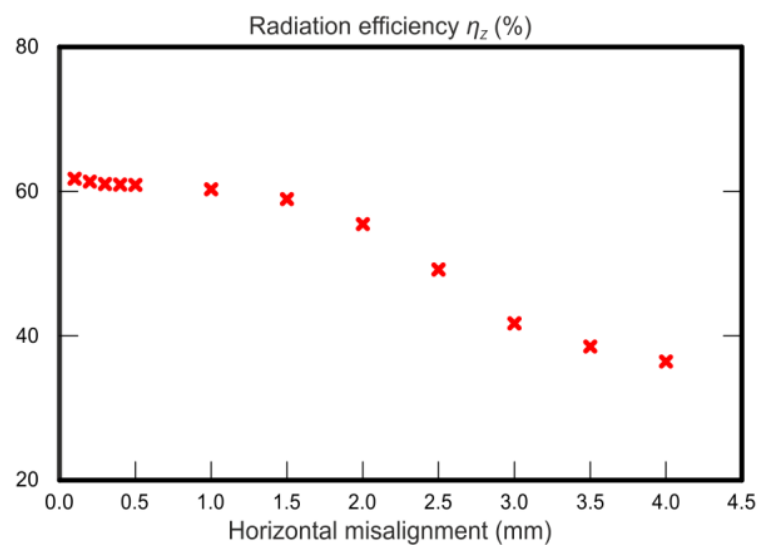

Fig. 4. Radiation efficiency of the transformer-antenna system, varying the horizontal misalignment between the windings for a constant tape thickness equal to $100 \mu \mathrm{m}$.

The radiation efficiency $\eta_{Z}$ (with respect to the project optimum impedance equal to $80-\mathrm{j} 103.2 \Omega$ at $2.45 \mathrm{GHz}$ ) as a function of the distance and the misalignment is plotted in Figs. 3 and 4, respectively. Its behavior is affected by the reflection coefficient, considering that $\eta$ does not change significantly when distance and misalignment vary. The efficiency $\eta_{z}$ decreases as a function of the misalignment but it remains quite constant for an offset up to $2 \mathrm{~mm}$. Yet, even for a large misalignment of $4 \mathrm{~mm}$, we still obtain an efficiency of
$36.5 \%$. The windings distance depends on the bi-adhesive tape thickness and this parameter can be controlled accurately. Meanwhile, the efficiency results to be robust with respect to the misalignment that depends on the alignment tolerance of the mounting process.

\section{RESULTS}

Finally the overall system antenna-transformer was fabricated, and measured. The prototype before and after the assembly is shown in Fig. 5. One contact of the one-turn primary winding realized on the patch ground plane is connected to the patch by means of a via, whereas the other contact is connected to the ground plane.

The radiation efficiency and the maximum gain, obtained from the simulation of the entire system, are equal to $-1.96 \mathrm{~dB}$ $(63.6 \%)$ and $6.49 \mathrm{dBi}$ respectively at $2.45 \mathrm{GHz}$. The maximum gain is similar to the results obtained by measuring other square patch antennas [2-4]. This means that the feeding topology does not significantly affect the antenna performance. Studies about the bending effects on patch antennas are shown in [2-4].

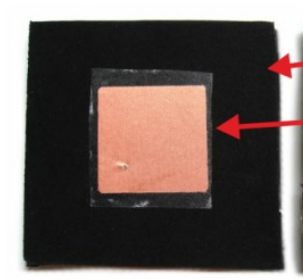

a

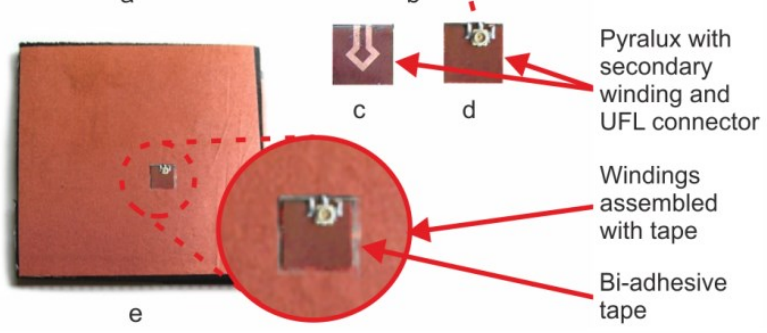

Fig. 5. Prototypes of the antenna-transformer: a) front view and b) back view of the antenna with the primary winding; c) top view and d) bottom view of the Pyralux square with the secondary winding and the UFL connector; e) assembled prototype with bi-adhesive tape. After assembly with bi-adhesive tape, the windings are not visible anymore (the primary is covered and the secondary is on the other side of the Pyralux square) and only the UFL connector is accessible (zoomed area).

The reflection coefficient $S_{11}$ was measured by means of the VNA and normalized with respect to the optimum load impedance, $Z_{L}$ OPT $=80-\mathrm{j} 103.2 \Omega$, obtained from the transformer EM simulation. The magnitude of the reflection coefficient remains below $-10 \mathrm{~dB}$ in the complete frequency band $2.4 \div 2.4835 \mathrm{GHz}$. The results are shown in Figs. 6 and 7 . The simulated and measured reflection coefficients are plotted and compared on the Smith chart with reference impedance of $50 \Omega$ in Fig. 6: the simulated results are obtained by simulating the overall antenna-transformer structure (blue line) and by cascading the two-port S-parameters of the transformer and the one-port S-parameters of the antenna (black line) simulated separately during their design. The 
magnitude of the measured reflection coefficient is plotted in Fig. 7 to verify the impedance bandwidth in the operating band.

A comparison of the present results with the State-of-Art (SoA) of textile patch antennas operating at $2.45 \mathrm{GHz}$ is summarized in Table II.

TABLE II

COMPARISON OF SOA

\begin{tabular}{|c|c|c|c|c|}
\hline Ref. & $\begin{array}{c}\text { Radiation } \\
\text { Efficiency (\%) }\end{array}$ & $\begin{array}{l}\text { Gain } \\
(\mathrm{dBi})\end{array}$ & $\begin{array}{l}\text { Tolerance to } \\
\text { misalignment } \\
\text { analysis }\end{array}$ & Mounting \\
\hline$[2]$ & - & 6.99 & Not available & $\begin{array}{l}\text { Soldering } \\
\text { process }\end{array}$ \\
\hline$[3]$ & $\geq 70 \%$ & $\geq 6.5$ & Not available & $\begin{array}{l}\text { Soldering } \\
\text { process }\end{array}$ \\
\hline$[4]$ & - & 6.83 & Not available & $\begin{array}{c}\text { Alignment } \\
\text { process }\end{array}$ \\
\hline $\begin{array}{l}\text { This } \\
\text { work }\end{array}$ & 63.6 & 6.49 & Available & $\begin{array}{l}\text { Alignment and } \\
\text { gluing process }\end{array}$ \\
\hline
\end{tabular}

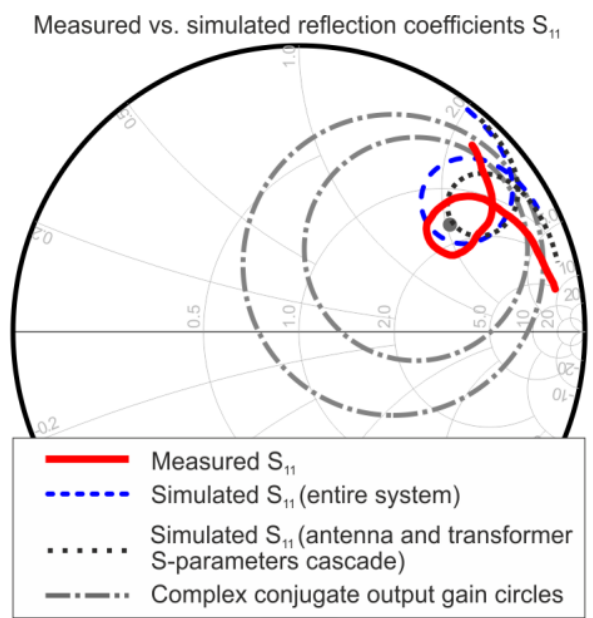

Fig. 6. Measurement vs. simulations of the antenna-transformer prototype: the reflection coefficients $S_{11}$ are plotted on the Smith chart with $50 \Omega$ reference impedance in the frequency range $2 \div 3 \mathrm{GHz}$. The complex conjugate of the load optimum impedance and of the gain circles (step of $-0.25 \mathrm{~dB}$ ) are obtained at $2.45 \mathrm{GHz}$.

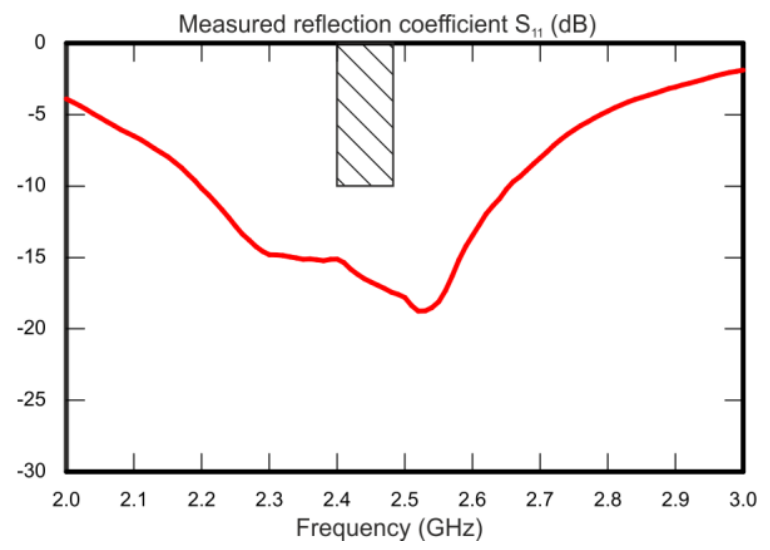

Fig. 7. Measurement of the antenna-transformer prototype: the reflection coefficient $S_{11}$ is plotted with respect to $80-\mathrm{j} 103.2 \Omega$ (optimal load impedance transformer).

\section{CONCLUSION}

A textile antenna with a transformer-based feeding topology, integrable into garments, was realized and measured. The coupling of the transformer windings avoids galvanic contacts between the antenna and the circuitry, allowing their isolation and passivation. The system operates in the ISM/SRD frequency band $(2.4 \div 2.4835 \mathrm{GHz})$, useful for Wi-Fi communication links in BAN. The ground plane reduces the interaction of the radiation with the human body. The simulations and the measurements show a good agreement and the system provides a measured reflection coefficient below $-10 \mathrm{~dB}$ in the overall operating frequency band. The results validate the use of this feeding topology, that can be implemented not only for patch antennas but also for other types of antenna. Moreover, the performed analysis about the effects of the windings misalignment on the system performance highlights its robustness.

\section{ACKNOWLEDGMENTS}

This work was carried out during the Short Term Scientific Mission (STSM) supported by COST RFCSET.

\section{REFERENCES}

[1] P. Hall, "Antennas and propagation for body centric communications," in Proc. IET Seminar Antennas and Propagation for Body-Centric Wireless Communications, London, U.K., Apr. 24, 2007, pp. 1-4.

[2] C. Hertleer, H. Rogier, L. Vallozzi, and L. Van Langenhove, "A Textile Antenna for Off-Body Communication Integrated Into Protective Clothing for Firefighters", IEEE Transactions On Antennas And Propagation, Vol. 57, No. 4, April 2009.

[3] A. Tronquo, H. Rogier, C. Hertleer, and L. Van Langenhove, "Robust planar textile antenna for wireless body LANs operating in $2.45 \mathrm{GHz}$ ISM band", Electronic Letters, 2nd February 2006, Vol. 42, No. 3

[4] C. Hertleer, A. Tronquo, H. Rogier, L. Vallozzi, L. Van Langenhove, "Aperture-Coupled Patch Antenna for Integration Into Wearable Textile Systems," Antennas and Wireless Propagation Letters, IEEE , Vol.6, pp.392-395, 2007.

[5] Hoi-Jun Yoo, "Advances in Textile-Based Electronics Are Weaving Computers Right into the Clothes We Wear", IEEE Solid-State Circuits Magazine, Mar. 2013, Vol. 5, Iss. 1, pp. 59-70.

[6] F. Alimenti, P. Mezzanotte, M. Dionigi, M. Virili, L. Roselli, "Microwave Circuits in Paper Substrates Exploiting Conductive Adhesive Tapes," Microwave and Wireless Components Letters, IEEE, Vol.22, No.12, pp.660-662, Dec. 2012.

[7] A. Finocchiaro, G. Ferla, G. Girlando, F. Carrara, and G. Palmisano, "A 900-MHz RFID system with TAG-antenna magnetically-coupled to the die," IEEE Radio Frequency Integrated Circuit Symposium, Atlanta (GA), USA, Apr. 2008, pp. 281-284.

[8] F. Alimenti, M. Virili, G. Orecchini, P. Mezzanotte, V. Palazzari, M. M. Tentzeris, L. Roselli, "A New Contactless Assembly Method for Paper Substrate Antennas and UHF RFID Chips", IEEE Transactions on Microwave Theory and Techniques, vol. 59, n. 3, March 2011, pp. 627 637.

[9] F. Alimenti, G. Orecchini, M. Virili, V. Palazzari, P. Mezzanotte, L. Roselli, "Design of Paper-Substrate Dipole Antennas Magnetically Coupled to UHF RFID Silicon Chips", IEEE International Conference on RFID-Technology and Applications, IEEE RFID-TA 2011, Sitges, Barcellona, Spain, 15-16 September 2011.

[10] Jerald Yoo, Seulki Lee, Hoi-Jun Yoo “A 1.12 pJ/b Inductive Transceiver With a Fault-Tolerant Network Switch for Multi-Layer Wearable Body Area Network Applications", IEEE Journal of Solid-State Circuits, Nov. 2009, Vol. 44, Iss. 11, pp. 2999-3010.

[11] "Alien Technology Corporation white paper: Fluidic self-assembly," Alien Technol., Morgan Hill, CA, 1999. [Online]. Available: http://www.alientechnology.com.

[12] Flectron ${ }^{\circledR}$ Metallized Materials Flexible metallized fabrics for a variety of EMI/RFI shielding applications [Online]. Available: http://www.lairdtech.com.

[13] DuPont Pyralux AP All-Polyimide Flexible Laminate [Online]. Available: http://www.dupont.com/fcm. 